\begin{tabular}{cc}
\hline & International Journal of Engineering \& Technology, $7(3.6)(2018) 9-12$ \\
SPC & International Journal of Engineering \& Technology \\
\hline
\end{tabular}

\title{
Automated Human Identification and Obstacle Avoidance for Visually Impaired
}

\author{
Priyanka Kumari ${ }^{1}$ \\ ${ }^{1}$ Student, SRM Institute of Science and Technology.
}

\begin{abstract}
This paper provides a method for human identification and obstacle avoidance for visually impaired. Visually impaired people faces lots of difficulty in accomplishing their day to day activities. Among one such difficulty is to recognize the person, this paper comes up with a technique which will helps blind people to identify person approaching them. Here DWT technique is used for face recognition. In this technique the entire image is decomposed into discrete wavelet bands. From this bands required features of image is obtained. This features when subjected to classifier gives proper output by identifying the person. Another part of paper deals with obstacle avoidance by using a blind stick. Blind stick uses an ultrasonic sensor and PIR sensor that detect obstacle at a distance of $100 \mathrm{~cm}$. This stick can be used as alert signal for blind people.
\end{abstract}

Keywords:DWT, classifier, obstacle avoidance, ultrasonic sensor, PIR sensor.

\section{Introduction}

Visually challenged people are among the boldest people existing on earthas they sense, smell and hear to live their life. They are confident enough that they have entered in field of government, teaching etc. Also, there is a lot of responsibility for them to lead their day-to-day activities with their authenticated persons [1]. The face identification system is gaining importance day by day. Military, biomedical and airport are some popular field where face identification is being used. The face identification system is also being used for security purpose [2]. The inherent properties of the human beings like pattern of fingerprint, face geometry, iris scanning are used as basic features for human identification. The face recognition based systems have a huge number of advantages over other system. For many other methods first step involves making bodily proximity with the system like exact position of iris and so on but here in case of FR a slight change in facial expression, this change does not affect working of the system [3]. Another part of paper deals with obstacle avoidance. Among many devices used white cane is the widely used among visually impaired. White cane is cheap, light weight and small so it can be folded and taken away in a pocket. However, users are taught for use of the white cane over periods of 100 hours. Many sophisticated devices, have been discovered and being used in the market for many years but lack usefulness, and as result they are not widely used [4]. In accordance with drawbacks of above technology, this paper provides a simple tool which can be easily affordable and widely available to blind people. A simple blind stick with ultrasonic sensor is used here and ArduinoUNO Microcontroller is also used.

\section{Methodology}

This paper uses methodology of discrete wavelet transform to recognize the image. The simulations are shown using MATLAB software. The algorithm used here is simple, first live image is taken from camera .This feature is extracted from image by DWT transform. Again corresponding database image is taken, the feature extraction of database image is done from image by DWT transform. Again corresponding database image is taken, the feature extraction of database image is done by DWT transform. Now the distance between features of both live image and database image is found out. If the distance is less than a particular value then the image is detected and if distance is more, image is not detected.

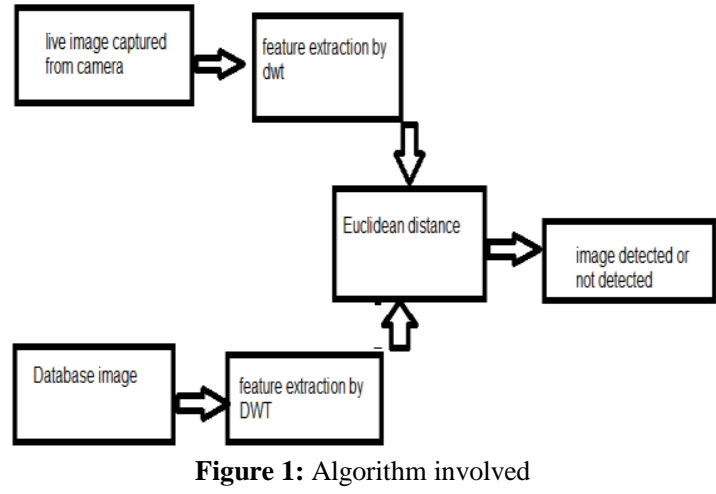

\section{Discrete wavelet transform}

DWT is one of popular transform used in image processing. Here in this paper we have used DWT technique for face recognition. It provides sub bands of image in terms of both frequency and time. The image in DWT is first passed through low pass filter and through high pass filter. Basic step in DWT is decomposition of entire image into sub bands, this process is called "resolution" of an image [2].

DWT are classified on basis of dimensions in which images are decomposed. DWT can one dimensional (1D), two dimensional (2D), and three dimensional (3D) [5]. $x[n]$ is an image signal and 
$\mathrm{g}[\mathrm{n}]$ is impulse response of filter through which signal $\mathrm{x}[\mathrm{n}]$ is passed.

$\mathrm{Y}[\mathrm{n}]=(x * g)[n]=\sum_{k=-\infty}^{\infty} x[k] g[n-k]$

\section{Euclidean distance}

In this paper we have used Euclidean distance as classifier to calculate distance between input image and database image. Distance between features of database and features of input image is found out. If the distance is less than certain value then image is detected. Basically is the distance between two points in any space. Suppose there are two points' $t$ and $r$, then the straight line connecting them is the distance between them.

Euclidean Distance $=\sqrt{ } \sum_{i=1}^{n}(t i-r i)^{2}$

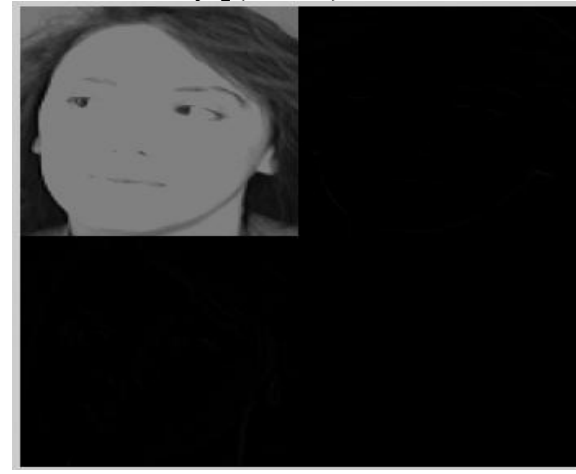

Figure 2: Images after DWT

Steps involved:

1. Video input of the image is taken by specifying particular resolution.

2. Video is given a name and is in RGB format.

3. Snapshot of the image immediately returns one single image frame and the snapshot taken is displayed.

4. Input file of the image is taken and read.

5. Size of image will be in matrix form.

6. If the color component of the image is 3 ,then convert RGB into grayscale else same grayscale image is taken.

7. The size of grayscale image is found out.

8. The DWT transform of image is taken.

9. Now the database features are loaded.

10. Distance between input image features and features of database image is found out. Distance value is $2.7 \mathrm{e}+004$.

11. If the value of distance is less than $3.9 \mathrm{e}+004$, then name of person is converted into audio file.

12. If the value is more, then the message displayed is "NOT DETECTED".

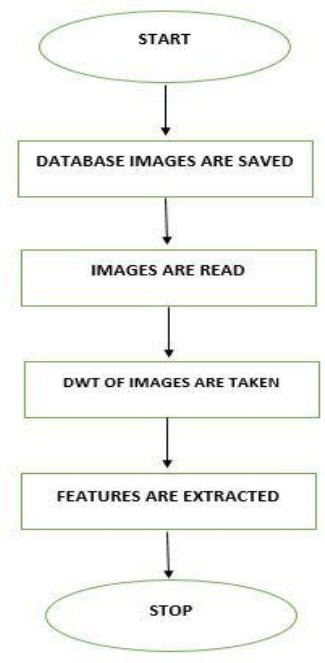

Figure 3: Algorithm involved for feature extraction of database images

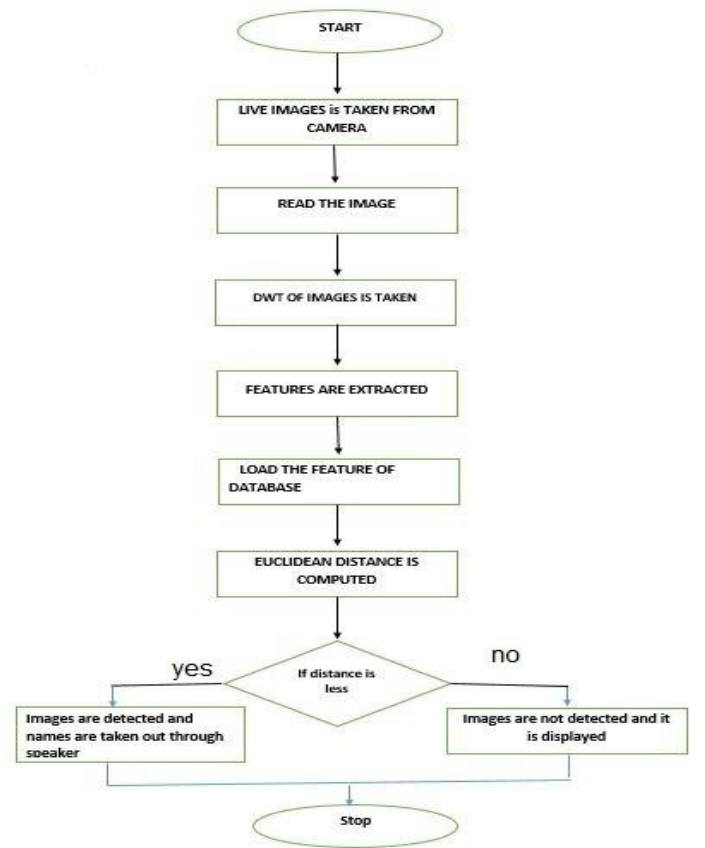

Figure 4: Algorithm involved for feature extraction and detection of live images

\section{Experiments conducted}

Face recognition is usually performed on standard database like ORL. Each image has resolution of $112 \times 92$. A training to testing ratio of $4: 6$ is used for experiments [5]. ORL type database were first one to be tested in laboratory and used for experiments [6]

A mixture of images in black and white and also FERET images are used. Each image has a different size and dimension

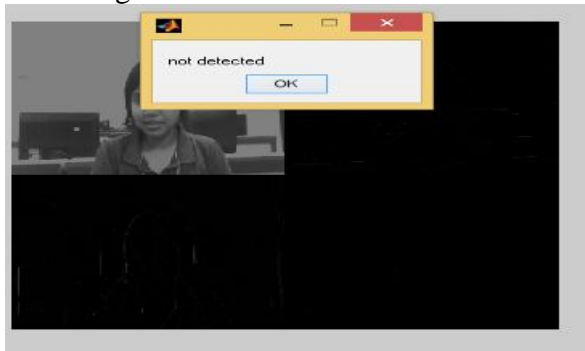

Figure 5: If images are not same as database image

\section{Training of database}

Training of the database is one of the most important steps in face recognition. Length of the folder containing database image is taken. All the images in database are formed in an array. The advantage of making it in an array form is that it can be easily compared. Now each image in database is checked for color components if the color component value is 3 then the data image is converted into gray scale else, the same image is taken. Now DWT transform is applied on the image. Features of the images are stored. Database contain sets of 14 images. All the images are trained one by one.

\section{Recognition time}

The total time for taking snapshots of live image through camera, extracting features through DWT transform and comparing both database and input images is 14.51027 seconds which is less than face recognition by DWT and Eigen vectors (53.98 s). So, this method could be proved to be more effective than other methods used. 
Here four faces are taken as input image from camera. Now the features extracted and corresponding time taken for recognition are tabulated.
a. Face 1 has dimension $640 * 480$.
b. Face 2 has dimension $1280 * 720$.
c. Face 3 had dimensions of $287 * 405$.
d. Face 4 had dimensions of $383 * 457$.

Table 1: Study of Features Extracted and Corresponding Time Taken

\begin{tabular}{|l|l|l|}
\hline Faces & Feature extracted & Time taken (in seconds) \\
\hline Face 1 & 3883 & 3.843 \\
\hline Face 2 & 8106 & 3.83 \\
\hline Face 3 & 7264 & 3.72 \\
\hline Face 4 & 3884 & 3.66 \\
\hline
\end{tabular}

\section{Obstacle Avoidance}

To avoid obstacle blind stick is designed. A blind stick has ultrasonic sensor to detect presence of objects or human near blind person. The smart kit consist of a stick with ultrasonic sensor HCSR04, a PIR motion detector sensor and an Arduino UNO board.

\section{Existing technology}

Previously many electronic devices were discovered to help blind people in performing their daily task, but this devices had lots of disadvantages and so they were not widely used[10]. Some of the devices used are:

\section{Electronic travel aid}

ETA are special devices that collect the happenings of surrounding world and transfer it to a system through sensors, actuators and many more $[9,10]$. Thus these devices help blind people in moving freely in their surroundings.

\section{Position locator devices}

This devices uses GPS technology to determine exact location of blind person.

\section{Ultrasonic headset}

Ultrasonic headset was used by visually challenged people. It consist of four types of ultrasonic sensor to analyze object in left and right orientation.

\section{Proposed scheme}

The proposed scheme main objective is to design a simple tool to detect obstacle for blind people. This tool will be easily affordable as well as easy to use. The main components required are:

\section{Ultrasonic sensor}

The sensor which is used here is ultrasonic sensor HC-SR04[11]. HC SR04 can be used in different types of processors like Raspberry pie, Arduino. The design of sensor is like they have two eye like piece. Here one eye is transmitter and other eye is receiver. The power is given to system by using vcc and ground pin. Input/output pin are ECHO and TRIG pin [4]

\section{Arduino Uno}

Arduino Uno is most popular microcontroller board. It is the system which processes analog input and gives us output in form of digital. Here programs are uploaded to detect obstacles in environment of blind person. Both input devices like sensor (ultrasonic and PIR sensor) and output devices (buzzer) is connected to it.

\section{PIR motion detector sensor}

The main purpose of using PIR sensor is that as a human being is detected buzzer sounds which will help blind people to understand that someone is standing in front of them.

\section{Algorithm}

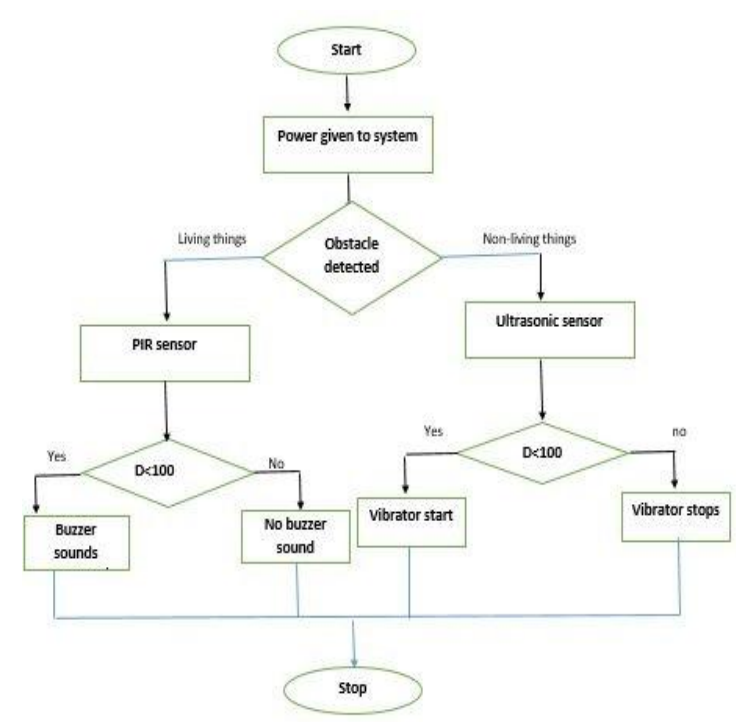

Figure 6: Algorithm for object detection

The algorithm for blind stick is very simple. Following steps are followed:

1. Power is given to system

2. Now an obstacle is detected by a sensor.

3. Ultrasonic sensor detects object if the distance is less than $100 \mathrm{~cm}$.

4. Buzzer sounds if object is detected by the sensor.

5. Living thing come across blind person then PIR sensor detects it.

6. Again buzzer sounds indicating that a human being is detected.

7. If the distance is more than $100 \mathrm{~cm}$ then object is not detected.

\section{Conclusion}

Thus a smart kit could be made which consist of a eye glass fitted with camera. The camera could take snapshots of surroundings and finally give the result. Smart kit also consist of a ultrasonic blind stick which can avoid obstacle at certain distance.

\section{References}

[1] Diwakar Srinath A, Praveen Ram AR, Siva R, Kalaiselvi VKG \& Ajitha G, "HOT GLASS Human face, object and textual recognition for visually challenged", 2nd international conference on computing and communication technology, (2017), pp.111-116.

[2] Koteswara Rao M, Veera Swamy K \& Anitha sheela K,"Face recognition using DWT and eigenvectors", 1st international conference on emerging technology trends in Electronics, communication \& networking, (2012).

[3] Mukhedkar MM \& Powalkar SB, "Fast Face Recognition Based on Wavelet Transform on PCA”, International conference on Energy system and Applications, (2015), pp.761-764.

[4] Chaurasia S \& Kavitha KVN, "An Electronic Walking Stick for Blinds", International conference on information communication and Embedded system, (2014), pp.1-5. 
[5] Yaji GS, Sarkar S, Manikantan K \& Ramachandran S, "DWT feature extraction based face recognition using intensity mapped unsharp masking and laplacian of gaussian filtering with scalar multiplier”, Procedia Technology, Vol.6, (2012), pp.475-484.

[6] Samaria FS \& Harter AC, "Parameterization of a stochastic model for human face identification", Proceedings of IEEE workshop on Applications of Computer Vision, (1994), pp.138-142.

[7] Roure J \& Faundez-Zanuy M, "Face recognition with small and large size databases", Proceedings 39th annual International carnahan conference on security technology, (2005), pp. 153-156

[8] Wafar E \& Elleithy K, "Sensor-Based Assistive Devices for Visually-Impaired People: Current Status, Challenges, and Future Directions", Ed. Panicos kyriacou sensors, Vol.17, No.3, (2017).

[9] Liu J, Liu J, Xu L \& Jin W, "Electronic travel aids for the blind based on sensory substitution", 5th International Conference on Computer Science and Education, (2010), pp.1328-1331.

[10] Sánchez J \& Elias M, "Guidelines for designing mobility and orientation software for blind children", Proceedings of the IFIP Conference on Human-Computer Interaction, (2007).

[11] Kanagaratnam K, "Smart Mobility Cane: Design of Obstacle Detection", EE 4BI6 Electrical Engineering Biomedical Capstones, (2009). 